\title{
Adaptive Method of Increase of Package Network Load
}

\author{
Alomar Saleh ${ }^{1}$, Alomar Mhamad ${ }^{2}$, V. M. Chuprin ${ }^{3}$ \\ ${ }^{1}$ Computer Engineering Department, Al-Ahliyyah Amman University, Ardhah, Jordan \\ ${ }^{2}$ Kyiv National University of Construction and Architecture, Kyiv, Ukraine \\ ${ }^{3}$ National Aviation University, Kyiv, Ukraine \\ Email: somar@ammanu.edu.jo,mr_moh_om@yahoo.com, vladimir@ndiasb.kiev.ua
}

Received April 22, 2013; revised May 22, 2013; accepted June 1, 2013

Copyright (C) 2013 Saleh Alomar et al. This is an open access article distributed under the Creative Commons Attribution License, which permits unrestricted use, distribution, and reproduction in any medium, provided the original work is properly cited.

\begin{abstract}
The method of loading increase of package networks is considered. This method is based on the dynamic redistribution of the throughput of switch equipment among carrying capacities (bandwidths) of its ports. Redistribution is fulfilled synchronously with the pulsations of packet streams, which move over the entrances of these ports. In the complement of switch equipment, it entered the adaptive mechanism of automatic real time redistribution of the throughput of switch equipment among the bandwidths of ports. This mechanism allows to decrease the quantity of time intervals, when intensities of packet streams are exceeded by the set bandwidths of ports, and, thus to increase the load of equipment by useful traffic.
\end{abstract}

Keywords: Bandwidths; Dynamic Redistribution; Throughput; Adaptive Mechanism; Package Networks

\section{Introduction}

Adaptive control by redistribution of resources of package networks is a type of dynamic management, and carried out in real time in accordance with the following principle: to the elements of network, loading on which presently approaches a critical point, greater part of network resources is selected due to diminishing of part of resources, which are selected by the under loaded elements. It is thus considered that the sum of all network resources during realization of management does not change and determine the total productivity of the involved equipment. Under network elements are understood, foremost, as a node equipment (in particular, package switches, routers and gateways), and also data links physically or logically. Under network resources are understood as the throughput of node equipment (package switches, routers, gateways and their ports) and (or) bandwidth of the physical (in Hertz) or logical (in a quantity of protocol data units (PDU), which are passed for time unit) data links. Terms of the "carrying capacity of port" and "bandwidth of port" in this work are considered as synonyms. Under load is understood as an amount of intensity of streams of package traffic, namely quantity of PDU, which passed on channel or processed node equipment for time unit. Loading factor of node equipment in the interval of supervision $\tau$ is determined by the relation of average intensity (average speed) of the treatment of PDU, attained on this interval $\tau$, to the throughput of node equipment. Loading factor of any separate port is determined by the relation of average intensity (average speed) of the treatment of PDU on this port to the carrying capacity (bandwidth) of this port. A typical example of using the principle of adaptive management: a multi-port router (or switch), whose capacity is redistributed between the strips ports in sync with changes in the intensity of packet streams that are transmitted through these ports.

A problem consisting of the traffic of modern package networks has pulsating poorly predictable character. The term of "pulsation of traffic" means rapid. Sometimes considerable changes are intensities of streams of protocol data units (PDU), what are going on in real time [1]. In the same time, the most known methods of traffic management in package networks are not used by the adaptive mechanisms of concordance of productivity of network equipment (package switches, routers, gateways and other) with current intensity of traffic in real time [2]. That is to say, the known mechanisms of traffic treatment do not provide dynamic changes of bandwidths of ports of switch equipment (SE) and, consequently, can't quickly change the parameters of tuning synchronously with the changes of current values of traffic pulsations. Absence of mechanisms of the dynamic retuning of parameters of 
SE ports (in particular, bandwidth of ports) results in appearance of good few of time intervals, when intensities of PDU streams are exceeded by the set values of carrying capacities of these ports. As a result, there is a substantial growth of coefficient losses of PDU. In such situation, to take possibility of network overload to the minimum, it is necessary to limit middle speed of PDU treatment on levels, substantially lower by comparison to the equipment productivity. So, it is necessary to work with the low values of network loading factor. For example, one of the most effective methods of increasing network load, known under the name "bucket of counters" [3], due to application of the special mechanisms of priority treatment and forming of traffic, is allowed in many cases to avoid the brief overload spades of network, but is not allowed to provide exploitation of equipment with a loading factor on levels higher 0.55 . Consequently, there is a ponderable reserve of increase of network load by useful traffic.

\section{General Raising of Task}

We will consider simple example of SE, which has three ports. We will consider that the general throughput of SE, marked as $H$, there is a sum of carrying capacities (bandwidths) of its ports $h_{1}, h_{2}, h_{3}$ :

$$
H=h_{1}+h_{2}+h_{3}
$$

We will also consider that a general throughput (1) is a constant, the choice of which is conditioned vehicle limitations. Intensities of package streams through SE ports (that, package traffics) will designate accordingly as $n_{1}, n_{2}, n_{3}$, will designate loading factors of ports as $k_{1}, k_{2}, k_{3}$, will designate reverse loading factors of ports accordingly as well as $\eta_{1}, \eta_{2}, \eta_{3}$ and we will define them as

$$
\eta_{1}=\frac{h_{1}}{n_{1}}, \eta_{2}=\frac{h_{2}}{n_{2}}, \eta_{3}=\frac{h_{3}}{n_{3}}
$$

Correlations (2) have simple physical maintenance: they show, as far as carrying capacities of ports of SE exceed intensities of package streams which served by them. Direct loading factors of ports are related to the reverse loading factors the followings correlations:

$$
k_{1}=\frac{n_{1}}{h_{1}}=\frac{1}{\eta_{1}}, k_{2}=\frac{n_{2}}{h_{2}}=\frac{1}{\eta_{2}}, k_{3}=\frac{n_{3}}{h_{3}}=\frac{1}{\eta_{3}}
$$

Direct loading factors (3) are shown by the levels of load of ports and can not be greater, than 1 . We will enter the vector of reverse loading factors of ports in consideration

$$
\dot{\eta}=\left(\begin{array}{l}
\eta_{1} \\
\eta_{2} \\
\eta_{3}
\end{array}\right)
$$

and vector of direct loading factors of ports

$$
\dot{k}=\left(\begin{array}{l}
k_{1} \\
k_{2} \\
k_{3}
\end{array}\right) .
$$

Vector form of record of expressions (4) and (5) simplifies the process of design of work of switch equipment at writing of the calculation programs.

Let current status of ports be characterized the set of their carrying capacities (that, by the set of their bandwidths), intensities of streams on the entrances of ports and proper direct and reverse loading factors of its ports. Let there is a mechanism of dynamic redistribution of bandwidths of ports in the direction of smoothing of loading factors of ports (Further this mechanism will be considered in detail). Then the process of adaptive control of the dynamic redistribution of bandwidths can be represented as equalizations of tuning:

$$
\begin{aligned}
& \dot{\eta}=f_{\eta} \text {-for reverse loading factors; } \\
& \dot{k}=f_{k} \text {-for direct loading factors. }
\end{aligned}
$$

Vector records of expressions (6) and (7) comfortably use for the construction of adaptive algorithm of retuning of SE in real time, which provide possibility of dynamic redistribution of carrying capacities of ports in connection with the possible pulsations of package traffics on these ports.

A type of right parts of equalizations of tuning will be represents below. While matters only that, as a result of "work" of equalizations of tuning (more precisely, as a result of process of adaptive control, which will be realized software and hardware facilities of SE), values of loading factors must become the same by the redistribution of carrying capacities of ports on condition of the main-tenance of their sum as constant - general unchanging throughput of switch equipment.

Taking into account foregoing will formulate the task of this research as follows. It is necessary to create the adaptive mechanism of the automatic redistribution of the throughput of switch equipment between carrying capacities (bandwidths) of its ports. This mechanism must provides possibility of dynamic change in real time of the bandwidth of ports [4], synchronously with changes pulsation intensity of PDU streams, which move over the entrances of these ports. It is necessary to make optimization of work of adaptive mechanism coming from the condition of maximization of loading factor of switch equipment at the set value of PDU loss coefficient. Thus the criterion of optimality must correspond with the condition of equality of loading factors for all ports for the stable mode, when intensities of streams, which move over ports, are permanent.

General scheme of work of adaptive redistributive mechanism. Functioning of adaptive mechanism must be 
carried out as follows. In periods of increase of stream intensity of PDU on some from ports an adjusting mechanism must select this port more considerable part from the general throughput of switch equipment [5]. Thus there must be the proper diminishing of carrying capacity (bandwidth) of port which diminishing (or constancy) of traffic intensity [6]. Dynamic changes of carrying capacities of ports, if they are executed synchronously with the current changes of traffic pulsations (in accordance with rules, which will be realized the mechanism of the adaptive adjusting), will provide diminishing of quantity of time intervals, when PDU stream intensities are exceeded by the set bandwidths of ports, and, thus, will increase a loading factor.

The structural scheme of adaptive control the bandwidths of ports of Switch Device (SD) is represented on Figure 1. As visible, the mechanism of automatic control is plugged in the subsystem of redistributing of carrying capacities of SD ports. This mechanism is provided by a management the bandwidths of SD ports so that the general throughput of SD was distributed between his ports in real time synchronously with changes intensity of PDU streams on SD ports.

Concordantly Figure 1 SD contains $\boldsymbol{n}$ ports, channels of access to which are conditionally represented as pointers. The current values of intensities of PDU streams on each of entrance ports of SD are measured facilities of measuring block of $M$. Measuring acts carried out consistently in time with a beforehand certain intervals. The results of measurements are given on the regulator of redistribution of carrying capacities of switch device ports $(R R C P)$. Also on every step of measurements information about the current sizes of bandwidths of all ports $\nabla \mathrm{F}$ moves on the regulator $R R C P$ from SD. The measured meanings of bandwidths were set on the previous step of iterative procedure of adaptive control. A regulator executes in real time iterative procedure of smoothing of current values of loading factors of ports so that the sum of these factors was saved unchanging. A smoothing algorithm gets out coming from

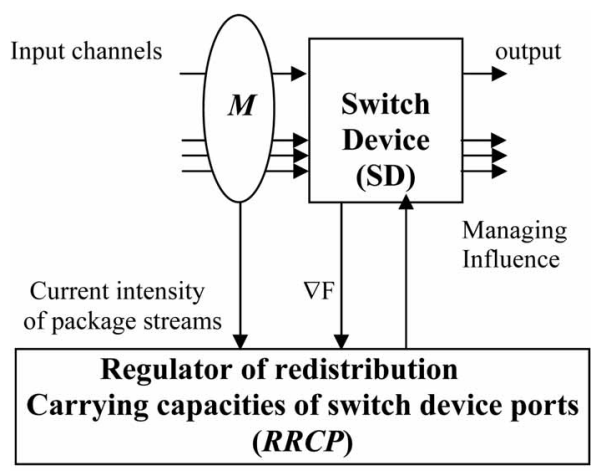

Figure 1. Scheme of adaptive control the bandwidths of switch device ports. the technical conditions of application of equipment. In any case the fast-acting of the adjusting system must be concerted with the parameters of pulsations of traffic. On every step of iterative procedure the count of the found new values of loading factors in the new values of bandwidths of SD ports is executed. Thus, on the output of regulator of $R R C P$ the stream of the managing affecting appears on executive mechanisms of the management system. These affecting initiate work of mechanism of redistribution of carrying capacities of ports.

Because of high dynamism and not complete predictable of character of changes speed of the real streams there is possibility of evaluation only of tendencies in changes speed on short time intervals. It is therefore suggested to use adaptive principle of construction of algorithm of automatic distribution of the throughput of switch equipment between carrying capacities (bandwidths) of its ports. The accepted law of redistribution must be represented through the proper differential equalization of tuning. Equalization of tuning must provide watching of tendencies in changes intensity of streams, and also to be a differential. Only the in this case formulated task in a raising aspect will be rich in content, reserved and inwardly no conflicting. Obvious useful investigation of its decision will be an increase of loading factor to at the set value of PDU losses coefficient.

General scheme of decision of task of adaptive control. Process of the dynamic redistribution of carrying capacity of ports at formal level it is possible to represent as differential equalization of tuning:

$$
\dot{\eta}=f_{\eta}
$$

where $\dot{\eta}$-vector of reverse loading factors of ports, and $f_{\eta}$-right part of tuning equalization, the type of which will be specified below. The values of loading factors must become the same by the redistribution of values of bandwidth each of ports upon condition of maintenance of their sum on permanent level. Numeral integration of tuning equalization on every step of adaptive control can be executed, for example, in obedience to the method of Euler:

$$
\dot{\eta}(t+h)=\dot{\eta}(t)+f_{\eta}(t) h,
$$

where $h$-step of numeral integration.

On the current step of integration have a new set of loading factors of ports, the values of which differ from each other on a less size, what it took place on the previous step of integration. The new recommended values of carrying capacities of ports, the sum of which is equal to the general throughput of switch equipment, corresponds the new values of loading factors of its ports. A general conclusion is such: for the decision of task of adaptive control of the bandwidths of switch equipment ports on the basis of looking in real time after changes intensity of package traffic on these ports it is necessary to decide the 
task of the dynamic smoothing of loading factors of switch equipment ports. Thus it comfortably uses some from the variants of method of the dynamic programming, which takes into account quality of transient process in the dynamically guided systems.

Thus, the task of the dynamic tuning of switch equipment is taken to the task of smoothing of loading factors of its ports. For its practical decision through the method of the dynamic programming it is necessary to specify tuning equalization, set an optimizing functional and write down the proper by it equalization of Bellman. Thus it is also necessary to set the type of function of Bellman. It will allow taking the task of the dynamic programming to the task of the analytical constructing of regulators, the decision of which, in same queue, is taken to the decision of equalization of Rikatti. All of it must be carried out "simultaneously", as all adopted higher fragments, which make maintenance of task of the analytical constructing of regulators, not exist separately, but must exactly correspond each other.

\section{Decision of Task the Method of the Dynamic Programming}

We will consider the task of smoothing of values a few the variables on condition of maintenance of their sum. Each of variables physically presents a size, reverse a loading factor of the proper switch equipment port. That, the values of loading factors become the same by the redistribution of carrying capacities of ports on condition of maintenance of their sum, equal the throughput of switch equipment. Let these variables form the vector of $N$. Will stipulate the conduct of components of vector by tuning equalization, specify the type of which as follows:

$$
\dot{N}=C \dot{u} \text {. }
$$

In equalization (9) $\grave{u}$-vector of managing influences, which is found as a linear function of components of vector $N$, and $C$-matrix of regulative interconnections, the quantity of columns in which corresponds the quantity of ports of switch equipment. The quantity of matrix lines corresponds the maximally possible number of pairs of ports, here in every pair the number of even one port differs from a number in any other pairs of ports. That, identical pairs are absent in the matrix of $C$, but all pairs are made from one great number of ports. The accepted laying out on pairs establishes regulative connections between all possible pairs of switch equipment ports. Thus the order of location of pairs in the matrix does not have of value.

For example, will consider Switch Device with three ports:

$$
\dot{N}=\left(\begin{array}{l}
n_{1} \\
n_{2} \\
n_{3}
\end{array}\right) \text {. }
$$

We will appoint the matrix of regulative connections of $C$ in the following kind:

$$
C=\left(\begin{array}{ccc}
1 & 0 & -1 \\
-1 & 1 & 0 \\
0 & -1 & 1
\end{array}\right)
$$

Between the first and third port regulative interconnection, which consists in that part of the throughput of SD is passed from the third port on first one, will be realized, that is reflected the proper signs of single elements of matrix. The second line accordingly reflects the transmission of part of the throughput of SD from the first port on third one. The third line reflects the transmission of the throughput of SD from the second port on third one. As visible, between three ports (as have three columns of matrix of $C$ ) it is possible in pairs to set three regulative interconnections that in the construction of matrix represented three lines. For the reflection of interconnections between four ports there must be six lines in the matrix of $C$, and between five ports are ten lines. We will mark possibility of ambiguous construction of matrix of $C$. In particular, columns and lines can be changed placed. The transposed record of matrix of $C$ is also possible.

We will find the type of function, suitable for constructing of regulator of $R R C P$ (look Figure 1). For this purpose we will present multiplication of vector (10) and matrix (11) in a kind

$$
\begin{aligned}
\dot{N}^{\mathrm{T}} C & =\left(n_{1}, n_{2}, n_{3}\right) \cdot\left(\begin{array}{ccc}
1 & 0 & -1 \\
-1 & 1 & 0 \\
0 & -1 & 1
\end{array}\right) . \\
& =\left(n_{1}-n_{2}, n_{2}-n_{3}, n_{3}-n_{1}\right)
\end{aligned}
$$

Multiplication (12) is presented by a transposed vector, that row-vector. Every component of row-vector is determined a difference between components of vector of $N$.

Further we will appoint the diagonal positively certain matrix of gravimetric coefficients, taking into account "weight" of every regulative interconnection:

$$
P=\left(\begin{array}{ccc}
p_{11} & 0 & 0 \\
0 & p_{22} & 0 \\
0 & 0 & p_{33}
\end{array}\right)
$$

And, finally, using a matrix (13), will build the following quadratic form:

$$
\begin{aligned}
& \dot{N}^{\mathrm{T}} C P C^{\mathrm{T}} \dot{N} \\
& =\left(n_{1}-n_{2}, n_{2}-n_{3}, n_{3}-n_{1}\right) \cdot\left(\begin{array}{ccc}
p_{11} & 0 & 0 \\
0 & p_{22} & 0 \\
0 & 0 & p_{33}
\end{array}\right) \cdot\left(\begin{array}{c}
n_{1}-n_{2} \\
n_{2}-n_{3} \\
n_{3}-n_{1}
\end{array}\right) \\
& =p_{11}\left(n_{1}-n_{2}\right)^{2}+p_{22}\left(n_{2}-n_{3}\right)^{2}+p_{33}\left(n_{3}-n_{1}\right)^{2} .
\end{aligned}
$$


As visible, expression (14) presents the self-weighted sum of squares of difference of variables, which constituents vector of $N$. comfortably to use expression (14) for a search the function of Bellman, and also optimizing functional, by which it is possible to take the task of the dynamic programming to the task of the analytical constructing of regulators. Expression (14) - it is positively certain and equals a zero only in the case when all variables equal a zero.

Will execute forming of optimizing functional taking to account that it is necessary to provide the set speed of process of smoothing of variables, and also a periodic (not shake) character of changes of the guided variables. For these terms optimizing functional $I$ comfortably to set in a kind

$$
I=\int_{0}^{\infty}\left(\dot{N}^{\mathrm{T}} C P C^{\mathrm{T}} \dot{N}+\alpha \dot{N}^{\mathrm{T}} C Q C^{\mathrm{T}} \dot{N}+\dot{u}^{\mathrm{T}} R \dot{u}\right) \mathrm{d} t,
$$

where $N$ is vector of the guided variables, $T$ is character of operation of transposition of matrix, $C$ is a matrix of regulative interconnections, $P$ is the $m \times m$-measured quadratic symmetric not negatively certain matrix of gravimetric coefficients, $\alpha$ - it is a positive constant (index of fading in function of Bellman), $Q$ is the $m \times m$ measured positively certain symmetric matrix of quadratic form (of function of Bellman), $\grave{u}$ is a vector of managing influences, $R$ is the $m \times m$-measured symmetric positively certain matrix of gravimetric coefficients at managements.

The first member of subinterval expression in a functional (15), as specified already, presents the selfweighted sum of squares of difference (through the coefficients of matrix of $R$ ) of the evened variables, which constituents vector of $N$. What this sum anymore or than longer smoothing process, the anymore value of optimizing functional. Therefore minimization of functional results in smoothing of the guided variables. The second member in a functional presents the function of Bellman. On optimum trajectories the Bellman-function decreases at a speed of not less, than multiplication of it and fading index $\alpha$. By the selection of value of $\alpha$-index, in the conditions of the closed system of adjusting, it is possible to set the desired speed of process of smoothing of the guided variables. In practice the index of fading of Bellman-function is used for the concordance of speed of work of mechanism of redistribution of carrying capacities of SD ports and current dynamics of pulsations of package traffic, acting on these ports. The third member of functional (15) determines the "conduct" of regulators, in particular limits a management and from the formal point of view locks procedure of determination of optimum management.

Will search the Bellman-function as a quadratic form:

$$
V=\dot{N}^{\mathrm{T}} C Q C^{\mathrm{T}} \dot{N} .
$$

Taking into account expressions for an optimizing functional (9) and set Bellman-function (16) equalization of Bellman will have the following kind:

$$
\begin{aligned}
0=\min _{\dot{u}}( & \dot{N}^{\mathrm{T}} C P C^{\mathrm{T}} \dot{N}+\alpha \dot{N}^{\mathrm{T}} C Q C^{\mathrm{T}} \dot{N} \\
& \left.+\dot{u}^{\mathrm{T}} R \dot{u}+\dot{N}^{\mathrm{T}} \frac{\partial V}{D \dot{N}}+\frac{\partial V}{D \dot{N}^{\mathrm{T}}} \dot{N}\right)
\end{aligned}
$$

If to put in equalization (17) expression for tuning equalization, will get:

$$
\begin{aligned}
0=\min _{u}( & \dot{N}^{\mathrm{T}} C P C^{\mathrm{T}} \dot{N}+\alpha \dot{N}^{\mathrm{T}} C Q C^{\mathrm{T}} \dot{N}+\dot{u}^{\mathrm{T}} R \dot{u} \\
& \left.+\dot{u}^{\mathrm{T}} C^{\mathrm{T}} C Q C^{\mathrm{T}} \dot{N}+\dot{N}^{\mathrm{T}} C Q C^{\mathrm{T}} C \dot{u}\right)
\end{aligned}
$$

By differentiation on the vector of management of expression (18) with the subsequent equating of the got result to a zero will define the vector of management of $\grave{u}$ as

$$
\begin{aligned}
& \dot{u}=-R^{-1} C^{\mathrm{T}} C Q C^{\mathrm{T}} \dot{N}, \\
& \dot{u}^{\mathrm{T}}=-\dot{N}^{\mathrm{T}} C Q C^{\mathrm{T}} C R^{-1} .
\end{aligned}
$$

The got management (193) is certain through the unknown quadratic form matrix of $Q$, entering in the complement of expression (16), which determines the Bellman-function. For determination of $Q$-matrix will put expression (19) in equalization of Bellman (18). After some transformations will get equalization of Rikatti in the following form:

$$
P+Q \frac{\alpha}{2} E+\frac{\alpha}{2} E Q-Q C^{\mathrm{T}} C R^{-1} C^{\mathrm{T}} C Q=0 .
$$

Deciding equalization (20), will find the sought-after $Q$-matrix. Putting the found matrix in expression (20) will get final expression for a regulator, which will have the following kind:

$$
\dot{N}=-C R^{-1} C^{\mathrm{T}} C Q C^{\mathrm{T}} \dot{N} .
$$

Analyzing expression (21), easy to notice that at any initial values of vector of the guided variables the adjusting system aspires to the stable state, when components of this vector is equal between itself, and the sum of values of components during all process of adjusting remains permanent. So set the problem it can consider decided.

It is important to mark that before the beginning of the use of SD it is necessary to co-ordinate speed of work of mechanism of redistribution of carrying capacities of SD ports with the current dynamics of pulsations of package traffic by the selection of value of index of fading of Bellman-function. In practice the index $\alpha$ is used for the concordance of speed of work of mechanism of redistribution of carrying capacities of switch equipment ports with the current dynamics of pulsations of package traffic, acting on these ports. 


\section{Basic Results and Conclusions}

1) Absence of mechanisms of the dynamic retuning of parameters of switch equipment ports in real time (in particular, retuning of bandwidths of its ports) in the conditions of considerable traffic pulsations results in appearance of good few of time intervals, when intensities of streams of protocol block units (PDU) are exceeded by the set values of carrying capacities of these ports. As a result, there are substantial losses of PDU even in the conditions of comparative to the small load of equipment by useful traffic. A method, which is based on the dynamic redistribution of the throughput of switch equipment between carrying capacities (bandwidths) of its ports in real time, can substantially increase the load of equipment and, at the same time, save the values of package loss coefficient on possible levels.

2) The structural scheme of adaptive control of the bandwidths of switch equipment ports is considered. In basis of scheme, the process of tracking after changing intensity of package traffic on ports of switch equipment in real time is fixed. The proper changes of the bandwidths of switch equipment ports are fixed so that more high intensity streams are taken considerable parts of the general throughput of switch equipment.

3) The formal task raising of dynamic redistribution of the general throughput of switch equipment of package networks between the carrying capacities (the bandwidths) of its ports in real time is executed. The general decision method of this task is offered. It is marked that its decision is taken to the decision of task of the dynamic smoothing of loading factors of switch equipment ports on condition of maintenance of their sum. It is also marked that in the process of decision it is necessary to execute the analytical constructing of adaptive regulators of bandwidths of ports in the dynamic mode taking into account quality of transient process of adjusting. It is for this purpose expedient to use one of varieties of method of the dynamic programming of Bellman.

4) In accordance with the chosen method of the dynamic programming, the type of Bellman's function is set and the proper equalization of tuning of the automatic control system is synthesized, which are allowed to get mathematical expression for an optimizing functional taking into account that it is necessary to provide the set speed of process of smoothing of variables, and also a periodical (not shake) character of changes of the guided variables. Further Bellman's equation is built. The control vector is found. The substitution of the found control vector in Bellman's equation is gotten equalization of Rikatti. Deciding Rikatti's equalization, after some mathematical transformations, final expression is gotten for a regulator in an analytical form. Useful property of the built regulator is exposed: For any initial values of the controlled variables, vector control system tends to be a steady state, the components of this vector are equal, and the sum of the components throughout the regulatory process remains constant, so that the task can be considered solved.

5) Decision results of dynamic redistribution task of basic resource of switch-to his throughput - can be used for the increase of load of package networks in the conditions of considerable pulsations of useful traffic.

\section{REFERENCES}

[1] A. Erramitti, P. Pruthi and W. Willinger, "Fast and Physically-Based Generation of Self-Similar Network Traffic with Applications to ATM Performance Evaluation," Proceedings of the WSC, Atlanta, 7-10 December 1997, pp. 997-1004.

[2] R. Bolla, "Dynamic Inter-Vehicle Communication Network for the Support of Real-Time Traffic Control," In: R. Bolla, F. Davoli and C. Nobile, Eds., Proceedings of 8th IFAC Symptoms on Transportation, Crete, June 1997, pp. 1108-1112.

[3] V. G. Olifer and N. A. Olifer, "Computer Networks, Principles, Technologies, Protocols," SPb, Piter, 2001, $672 \mathrm{p}$.

[4] F. Xue, J. Liu, Y. Shu, L. Zhang and O. W. W. Yang, "Traffic Modeling Based on FARIMA Models," CCECE99 Proceedings, Edmonton, 9-12 May 1999, pp. 162-167.

[5] M. Ghaderi, "On the Relevance of Self-Similarity in Network Traffic Prediction," 2003.

http://www.cs.uwaterloo.ca/cs-archive/CS-2003/28/TR-C S-2003-28.pdf

[6] I. Norros, "The Management of Large Flows of Connectionless Traffic on the Basis of Self-Similar Modeling," IEEE International Conference on Communications (ICC'95), Seattle, 18-22 June 1995, pp. 344-356. 